\title{
DE LA TEORÍAA LA PRÁCTICA. EL PROCESO DE DISEÑO E IMPLANTACIÓN DE LOS GRADOS DE GEOGRAFÍA EN LAS UNIVERSIDADES ESPAÑOLAS
}

\author{
Javier Esparcia Pérez \\ Departamento de Geografía, Universidad de Valencia \\ Dolores Sánchez Aguilera \\ Departamento de Geografía Física y Análisis Geográfico Regional, Universidad de Barcelona
}

\section{RESUMEN}

La adaptación al Espacio Europeo de Educación Superior está suponiendo la penúltima fase de un largo proceso de cambios en la Geografía universitaria en España. Más que en otras reformas previas, los Grados suponen varios retos importantes para la comunidad geográfica. El más importante de estos es el de diseñar una formación menos centrada en los conocimientos y mucho más en el aprendizaje de habilidades y destrezas por parte de los estudiantes, menos en el profesor y más en el alumno. Todo ello persigue mejorar de las oportunidades de empleabilidad de los estudiantes. Sin olvidar algunas inercias, en este trabajo se hace un análisis del proceso de implantación de los nuevos Grados de Geografía en España.

Palabras clave: Planes de estudios, Espacio Europeo de Educación Superior (EEES), Grado, Geografía.

\section{ABSTRACT}

The adaptation to the European Higher Education Area is being the penultimate phase of a long process of change in the academic Geography in Spain. More than in other previous

Fecha de recepción: enero 2012.

Fecha de aceptación: febrero 2012. 
reforms, the new Degrees involve several major challenges to the geographic community. The most important of these is to design a system less focused on knowledge and much more on learning abilities and skills by students, less on the teacher and more on the student. All this aims to improve opportunities for students' employability. Without forgetting some inertia that still remains, this paper provides an analysis of the process of implementation of new Degrees in Geography in Spain.

Key words: Curricula, European Higher Education Area (EHEA), academic degrees, Geography.

\section{ANTECEDENTES: LA EVOLUCIÓN DE LAS LICENCIATURAS EN GEOGRAFÍA}

En los últimos años el sistema universitario español está inmerso en el proceso de adaptación al Espacio Europeo de Educación Superior (EEES). Completar adecuadamente esta adaptación es un reto aún vigente para el sistema universitario, pero hacerlo con éxito lo es también para la sociedad española. Este proceso está suponiendo, en primer lugar, una oportunidad para superar muchas inercias del sistema universitario, pero también ineficacias demostradas en nuestra respuesta a las necesidades sociales. En segundo lugar, y de manera paralela, este proceso ha constituido una doble oportunidad, por un lado para modernizar las estructuras del principal instrumento docente del sistema universitario, los planes de estudio, y adaptarlas a la nueva situación; y por otro, para incorporar y consolidar nuevos enfoques en el proceso de enseñanza-aprendizaje, que hagan de los nuevos títulos un instrumento eficaz a la hora de formar profesionales. Con todo ello, el objetivo último es que el sistema universitario sea capaz de responder más y mejor a las necesidades sociales.

Estos son retos que afectan a todo el sistema universitario español en general. Pero hay algunas disciplinas para las que, como es el caso de la Geografía, por sus características y trayectoria, estos retos son especialmente importantes. Efectivamente, la Geografía, siendo una de las disciplinas más antiguas, es a su vez una de las ciencias más modernas. Y es posible que esto último esté en la base de que, entre otras dificultades y a diferencia de muchas otras disciplinas, afronte aún hoy un verdadero déficit de legitimidad social. Los geógrafos no siempre hemos sido - o querido ser - suficientemente conscientes de esta dificultad, y de ahí que nos hayamos debatido entre el inmovilismo académico (probablemente ayudados por la comodidad de unas estructuras universitarias con frecuencia bastante alejadas de la sociedad), y los esfuerzos por hacer de nuestra disciplina una ciencia cada vez más presente, relevante y útil socialmente.

La Geografía ha tenido una evolución relativamente rápida en este último medio siglo (Burriel de Orueta, 2004a, 2004b). Desde la antigua licenciatura de Filosofía y Letras, la reforma de 1973 supuso la implantación de una licenciatura en Geografía e Historia, con una especialidad en Geografía en los dos últimos años. Posteriormente, la reforma de los planes de estudio de los años 90 constituyó un primer esfuerzo por introducir cambios de estructuras y procedimientos que permitiesen avanzar en ese proceso de modernización, tan necesario. Había una situación caracterizada, en primer lugar, por la necesidad de supervivencia de la nueva licenciatura en Geografía, y en segundo lugar por un perfil de alumnado en el que 
predominaban las segundas, terceras y hasta cuartas opciones. Con la minirreforma del año 2000 se avanza hacia una perspectiva con un carácter más profesional y aplicado, en torno en gran parte a la Ordenación del Territorio. Esta fue una de las innovaciones más significativas, a lo cual contribuyó el que confluían diferentes disciplinas y sobre todo el que había una demanda y una necesidad social de la ordenación del territorio. Por otro lado en estos años, se abría la puerta hacia la renovación de estrategias docentes, que en su caso habían de ser la verdadera muestra del esfuerzo en cada disciplina o de cada grupo de profesionales a la hora de adaptarse a los nuevos retos, siempre dentro del margen de maniobra que podía permitir el sistema universitario (recursos humanos y materiales disponibles, tamaño de los grupos, horas lectivas, enfoque y procesos de aprendizaje, etc.).

En lo que respecta a la disciplina de la Geografía (separada de la tradicional licenciatura de Geografía e Historia desde los primeros años 90), uno de los elementos más característicos fue una cierta convergencia hacia esa vertiente más aplicada de la Geografía (aunque a efectos prácticos este no ha sido un proceso unánime), la profundización en contenidos (especialización), y la creciente importancia de las materias técnicas ${ }^{1}$. Ello no evitó, pese a los vínculos entre Geografía y ordenación del territorio, que el descenso en la matrícula continuara desde mediados o fines de los años 90 (Esparcia y Sánchez Aguilera, 2009, 2010). En ese contexto, se habían producido algunos avances, modestos en todo caso, en la definición de perfiles profesionales; y debido probablemente a esos avances la inserción profesional de los geógrafos fue mejorando, de manera más destacada en algunos ámbitos (Colegio Profesional de Geógrafos, 2008).

Efectivamente, la inserción profesional es probablemente el mejor indicador respecto de unos planes de estudio adaptados a las posibilidades profesionales y las necesidades sociales (Arrowsmith et al., 2011) ${ }^{2}$. En España se han realizado algunos análisis sobre los resultados de todo aquel proceso de los años 90, en aspectos como la inserción profesional de los licenciados, que es sin duda el indicador más claro del nivel de éxito de una disciplina (siempre ponderando adecuadamente los resultados con el grado de dinamismo global en el mercado de trabajo). Así por ejemplo el Libro Blanco de Geografía y Ordenación del Territorio (ANECA, 2004) ponía de relieve algunos de estos avances en la inserción profesional de los licenciados en Geografía, fruto de esa orientación profesional pero también del contexto expansivo que la ordenación del territorio ha venido teniendo en nuestro país en estas últimas décadas. Y es en este contexto en el que han experimentado una destacada expansión áreas como las tecnologías de la información geográfica o el medio ambiente. Pero si desde los primeros años 90, y sobre todo durante la última década, se han formalizado unos perfiles profesionales ligados a la Geografía, sigue pendiente mejorar tanto la visibilidad social de esos perfiles profesionales como de su competitividad con relación a la satisfacción de las demandas y/o necesidades sociales.

1 Esta reorientación hacia una visión más aplicada se tradujo también en el cambio parcial de nombre de algunos departamentos de Geografía que, con ello, pretendían poner de relieve el énfasis en esa vertiente aplicada.

2 Para una discusión sobre las diferentes concepciones de la empleabilidad de los geógrafos puede verse el análisis comparativo de Rooney et al. (2006), en el que se analiza la situación en Italia, Reino Unido, Estados Unidos, Chile, Estonia, Grecia y España. A partir de la constatación de que los estudiantes de geografía tienden a tener niveles elevados de empleabilidad, se analiza la importancia que en los currícula se le da a este objetivo para los estudiantes de geografía en cada uno de los casos estudiados, poniendo de relieve algunas diferencias significativas. 
Por tanto, esos avances en la inserción profesional se han ido logrando pese a que no se habían diseñado programas formativos y procesos de enseñanza-aprendizaje suficientemente adaptados a esas demandas y/o necesidades sociales. Este seguía siendo un gran reto para la disciplina.

Y un segundo reto estaba en el lado de la formación universitaria. No disponemos de análisis ni tan profundos o sistemáticos en lo que respecta a los cambios que se introdujeron en los procedimientos de aprendizaje-enseñanza y su impacto sobre los estudiantes. Pero todo parece indicar que los avances en este ámbito han sido mucho menos significativos. Por ello el EEES constituye una oportunidad tanto para profundizar en aquellos cambios como, en su caso, para rectificar algunas de las deficiencias ${ }^{3}$. Y precisamente en estos momentos, en los que la mayor parte de universidades están a punto de finalizar el proceso de implantación de los Grados, es ya un buen momento para reflexionar sobre las ventajas, inconvenientes y aspectos pendientes en la introducción de este nuevo marco para nuestra disciplina.

Y aunque es ya tradicional, sigue siendo necesaria una serena reflexión sobre la función de la Geografía en este nuevo escenario que es el EEES y con este nuevo instrumento que son los Grados, pero sobre todo una reflexión más profunda y casi permanente sobre qué función queremos y/o podemos adoptar los geógrafos en el doble contexto del nuevo mapa de titulaciones, por un lado, y del entorno social y territorial, por otro. Este proceso obliga a la Geografía universitaria, pero también a los geógrafos profesionales no universitarios, a una doble reflexión. En primer lugar, nuestra inserción en ese mapa de titulaciones y el lugar que podemos y/o deseamos ocupar respecto de las necesidades y demandas sociales, y hasta qué punto la experiencia de implantación de los Grados responde a ese margen de maniobra y a ese deseo del colectivo. Y en segundo lugar, es necesario mantener la reflexión sobre cómo podemos y/o deseamos profundizar, en su caso, en la instauración, fortalecimiento y mejora de los procesos de enseñanza-aprendizaje que requiere la implantación plena y consolidación de las nuevas titulaciones. Y este es un esfuerzo que no finaliza con el diseño, y en su caso, implantación de los planes de estudio, sino que ha de ir mucho más allá, y mantener una reflexión permanente sobre el desarrollo curricular de la Geografía ${ }^{4}$.

Según este contexto, la Geografía, pese a definirse como Ciencia del Territorio y mantener una posición — de síntesis, según algunos - entre las humanidades, las ciencias sociales y las ciencias de la tierra (y probablemente debido también a ello), está inmersa en una situación crítica (aunque bien podría decirse que en realidad apenas hemos salido de esa situación

3 Para un análisis detallado del proceso de adaptación al EEES, así como de las dificultades del modelo español frente al modelo predominante en Europea, véase el excelente trabajo de Tulla (2010).

4 Una muy interesante reflexión sobre los currícula geográficos en el ámbito anglosajón la tenemos en Whalley et al., 2011. Los autores insisten en la necesidad de aportar destrezas orientadas a la empleabilidad como parte de unos muy cambiantes requisitos en el entorno socioeconómico y territorial, en el que han de integrarse el conocimiento y las destrezas geográficas. Y los geógrafos hemos de estar atentos a la necesidad de introducir cambios en los conocimientos impartidos, como por ejemplo adaptación y respuesta a los cambios ambientales, pero paralelamente hemos de poner a punto las destrezas necesarias en la perspectiva de la empleabilidad de los estudiantes. Ello implica, según los autores, que el profesorado diversifique los métodos docentes, orientándolos hacia un aprendizaje activo y en el marco de aproximaciones «problem-oriented». Véanse también a este respecto los trabajos de Hennemann et al., (2010), sobre el necesario balance entre conocimientos y competencias (a partir del estudio de grados de Geografía en Alemania), y Conway-Gómez et al., (2011), que se centran en métodos de enseñanza basados en la creación de sinergias. 
crítica en las últimas décadas). En todo caso, sigue siendo una disciplina-ciencia aún poco comprendida, y menos aún compartida, por muchos doctos profesionales no geógrafos, y obviamente sigue siendo poco conocida en lo que son las potencialidades reales. Por ello el déficit de legitimidad social, que es innegable pese a los avances en estas últimas dos décadas, va parejo a la necesidad de una mayor legitimidad científica. Y obviamente el sistema universitario tiene ahí una importante responsabilidad, en la medida que esta mayor necesaria legitimidad científico-social pasa por una formación rigurosa de profesionales capaces de trabajar junto a otras disciplinas, dando respuestas a las importantes necesidades y demandas sociales sobre problemáticas territoriales.

Las potencialidades de la Geografía como disciplina consolidada socialmente dependen en gran parte de cómo los geógrafos, académicos y no académicos, seamos capaces de combinar y actuar en tres factores o ámbitos estratégicos:

a) En primer lugar hay que empezar por nosotros mismos, por el profesorado, por nuestra preparación y nuestro grado de compromiso con los retos asociados a la implantación y consolidación del Grado. Mayoritariamente el profesorado de geografía proviene aún de la licenciatura de Geografía e Historia, e incluso de Filosofía y Letras. Y aunque no cabe duda que ha habido una especialización y actualización importante en contenidos, no podemos decir lo mismo respecto de lo que suponen los procedimientos. En todo caso, también es cierto que es variable el nivel de compromiso con el reto que supone la implantación del Grado y los avances hacia nuevos procesos de enseñanza-aprendizaje.

b) El segundo factor lo constituyen precisamente esos nuevos procesos de enseñanzaaprendizaje orientados a la adquisición de competencias. Y aquí el EEES es, o pretende ser, mucho más que una simple reforma para adaptar la licenciatura de cinco a los grados de cuatro años. Es una reforma en la que al sistema universitario se nos pide que seamos capaces de desarrollar procedimientos, métodos y técnicas que permitan mejorar el aprendizaje de los alumnos, con un objetivo claro, su preparación óptima para el mundo profesional. Y por esta razón es responsabilidad del sistema universitario, por un lado, una buena detección de cuáles son las necesidades y demandas sociales a las que podemos y debemos dar respuesta. Por otro, es necesario trasladar adecuadamente tales necesidades a objetivos formativos claros, diseñando y poniendo a punto los instrumentos básicos para alcanzarlos, principalmente el Grado y los Masters; y aquí incluimos el esfuerzo que debe partir del propio estamento docente a la hora de introducir los mecanismos de reciclaje necesarios para que todo ese esfuerzo sea realmente eficaz, y no nos quedemos con simples cambios de títulos y/o reordenación de contenidos. Y finalmente, el sistema universitario, en conjunción con las administraciones públicas, tienen la responsabilidad de poner a disposición del propio sistema, del profesorado, los instrumentos de apoyo necesarios para aplicar esos procesos con la máxima eficacia.

c) El alumnado es el tercero de los factores estratégicos. Tradicionalmente la Geografía ha sido refugio de segundas, terceras y hasta cuartas opciones para los estudiantes. Ello supone que el estudiante que suele ingresar en Geografía no solo no la tiene entre sus preferencias, sino que no tiene una idea precisa de en qué consiste y qué se va a encontrar, y todo tiene como consecuencia una actitud, inicial al menos, poco 
receptiva. Pero con el Grado en Geografía ha aumentado el número de estudiantes de primera opción, y ello implica un cambio importante, porque estamos ante estudiantes con expectativas claras respecto de la Geografía, y dar respuesta a esas expectativas constituye un nuevo reto para el profesorado, un reto que exige, en parte, un cierto cambio de mentalidad y hasta de actitud respecto de cómo abordar los procesos enseñanza-aprendizaje en este nuevo contexto, basado no tanto en la adquisición de contenidos sino, sobre todo, de competencias. Los trabajos de Hennemann et al., (2010), y Whalley et al. (2011) son una pequeña muestra de cómo nuestros colegas geógrafos anglosajones abordan la enseñanza de la Geografía con la mirada puesta precisamente en el aprendizaje de competencias por parte de los estudiantes.

A continuación hacemos un breve repaso del proceso de construcción de las nuevas titulaciones de Geografía, de cómo está siendo el proceso de implantación en la Geografía española, y finalmente retomaremos algunos elementos de reflexión a la luz de la situación actual.

\section{LA CONSTRUCCIÓN DE LAS NUEVAS TITULACIONES: EL DISEÑO DE LOS GRADOS EN GEOGRAFÍA}

El diseño de los nuevos grados reabrió algunos debates en el seno de la Geografía española sobre la orientación profesionalizadora y el esfuerzo de reflexión que representó la elaboración del Libro Blanco de Geografía y Ordenación del Territorio (ANECA, 2004) sirvió como referente de las nuevas titulaciones. La mayor parte de grados son titulaciones que aúnan Geografía y Ordenación del Territorio, con las excepciones de la Universidad de Barcelona, Salamanca e Illes Balears, que tienen un grado de Geografía, la de la Universidad de Valencia, que ha orientado su grado hacia la Geografía y Medio Ambiente, y la de Girona, que plantea un grado mixto con estas dos grandes orientaciones profesionales, la ordenación del territorio y el medio ambiente Sin embargo, los nuevos títulos - caracterizados por el cambio de orientación y la incorporación de la ordenación como elemento vertebradordeben afrontar como el reto de dotar de contenido real ese perfil profesional (Prieto, 2010), y que el título de los grados no quede en una simple operación de marketing académico.

\subsection{La formación básica en los Grados de Geografía: el peso de las inercias tradicionales}

Un elemento novedoso de los grados era la necesaria adscripción de la titulación a una rama de las disciplinas científicas. La Geografía, situada en una encrucijada entre las ciencias sociales, las ciencias naturales y las propias humanidades, basculó entre incorporarse a la rama de Arte y Humanidades (donde el Real Decreto 1393/2007 de Ordenación de las Enseñanzas Universitarias la ubicaba) y en la de Ciencias Sociales y Jurídicas (que se incorporó como posibilidad de adscripción tras las protestas de diversos colectivos geográficos).

Las orientaciones de ANECA y la voluntad de desmarcarse del bloque de Humanidades hicieron que muchos departamentos elaboraran propuestas de Grado enmarcadas en la rama de Ciencias Sociales y Jurídicas. Este cambio de actitud responde a una cierta concienciación en el sentido de que a la Geografía le corresponde una posición entre las Ciencias Sociales, más que entre las Humanidades. Asimismo, la adscripción a la nueva rama debería desem- 


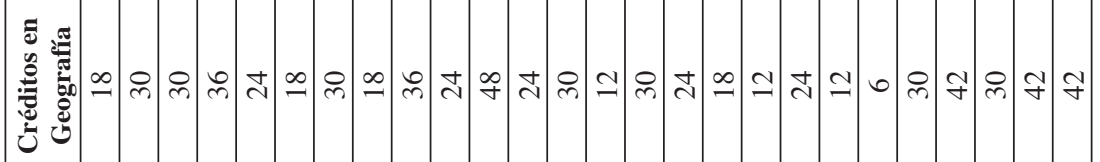

je

压

这

W

$\approx$

龺

苁

空

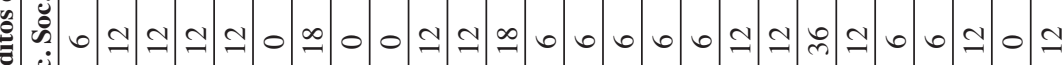
茪

$\stackrel{k}{\Sigma}$

20

毠

을



잉

능

(ำ

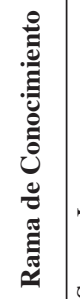

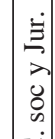

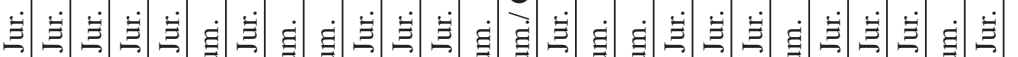

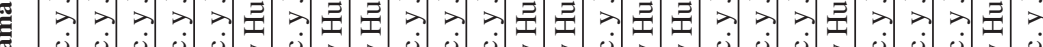



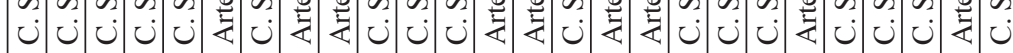

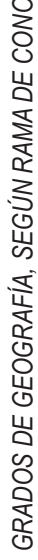

F

三ิ



完

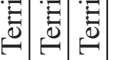

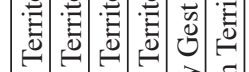

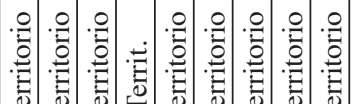



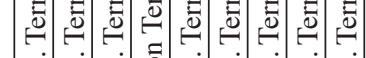

है

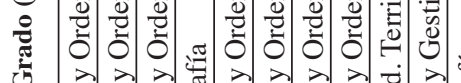

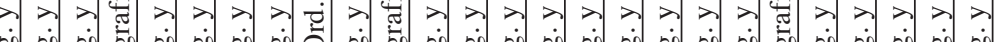

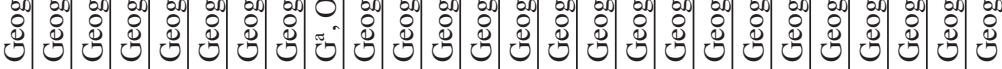

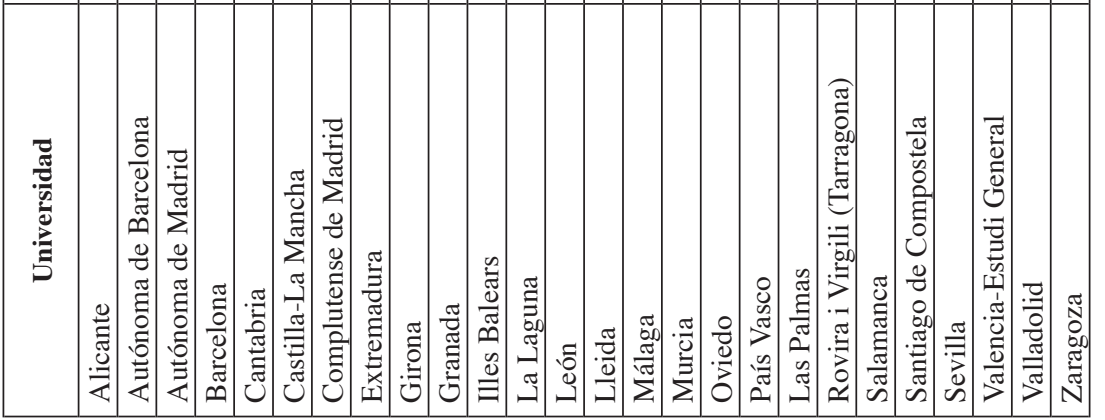


peñar un papel importante en otro de los elementos nuevos en el diseño de los grados, el referido a la definición de las materias fundamentales.

Sin embargo, la incorporación de créditos desde las disciplinas de ciencias sociales como formación básica (que supone la existencia de una notable transversalidad dentro de la misma rama de conocimiento) no se materializa de manera efectiva, de modo que muchos grados que se incluyen en la rama de Ciencias Sociales y Jurídicas tienen una proporción menor de créditos de materias de esta rama que de la de Arte y Humanidades (Tabla 2).

Por tanto, se observan cambios de actitud y una conciencia clara sobre la necesidad de reposicionar a la Geografía en un nuevo contexto, pero la lentitud de las transformaciones y el peso de las inercias - la tradición de vínculo con las humanidades y la misma ubicación física en muchos casos en facultades de Letras, de Filosofía y Letras o bien de Geografía e Historia - siguen condicionando los cambios reales, ya en la fase misma de diseño de los propios grados.

Un análisis de la práctica totalidad de Grados pone de relieve que casi el $45 \%$ de los créditos considerados como formación básica son materias de Geografía (Fig. 1), y esta es una tendencia bastante extendida (Tabla 2 y Fig. 2). Ese volumen de materias de Geografía incluido en la formación básica responde, en parte a la consideración del carácter de las asignaturas (fundamentales para la formación de un graduado en Geografía), pero también, en otros casos, a la idea de captar créditos - habida cuenta de la elevada capacidad docente en muchos departamentos y la necesidad de absorber el excedente de fuerza docente - por

Figura 1

DISTRIBUCIÓN DE LOS CRÉDITOS DE LAS MATERIAS CONSIDERADAS COMO FORMACIÓN BÁSICA (EN \%) EN LOS GRADOS DE GEOGRAFÍA

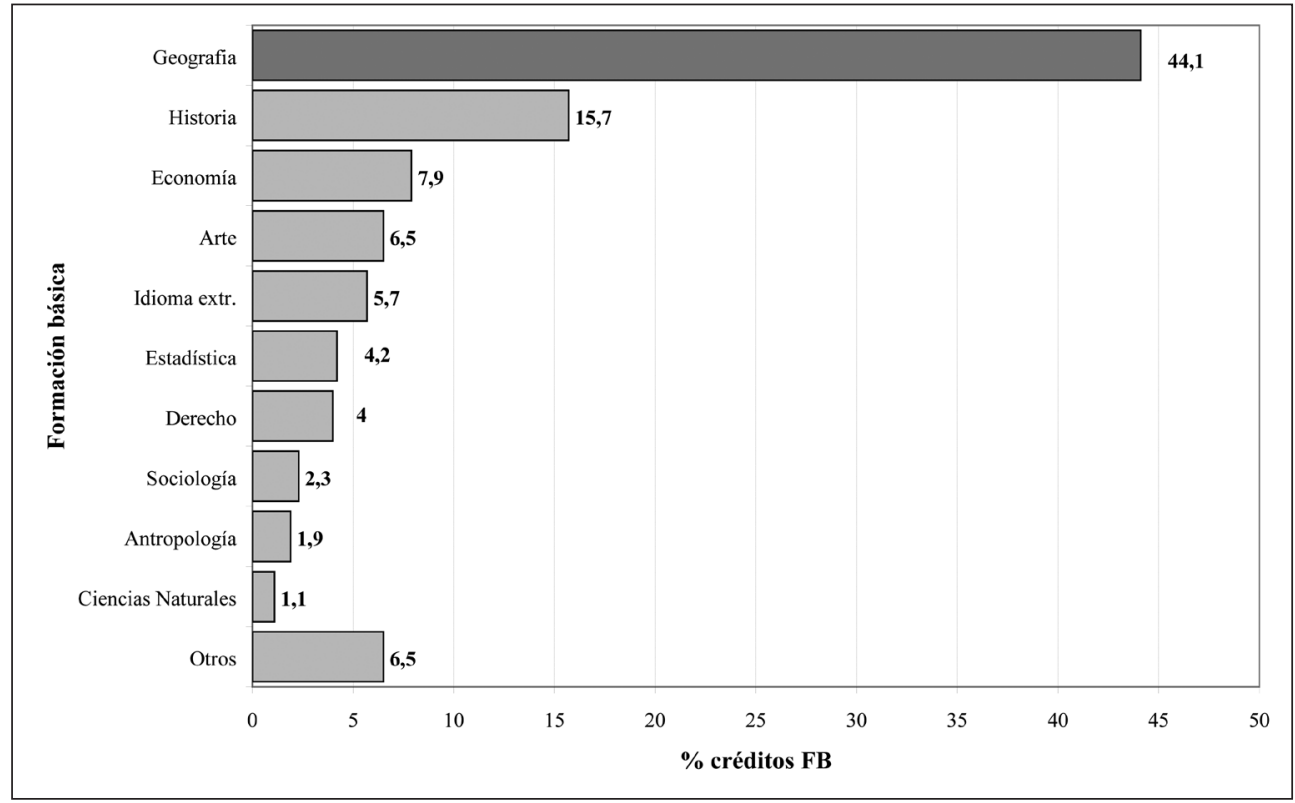

Fuente: Elaboración propia a partir de los grados de Geografía de las diferentes Universidades. 
lo que se trasladan materias que estaban en los primeros cursos de la licenciatura al nuevo contenedor de formación básica.

Por otra parte, una parte significativa de las materias no geográficas que componen la formación básica está constituida por asignaturas de materias de Historia y Arte, así como de idiomas extranjeros. Por el contrario, los créditos dedicados a disciplinas claramente enclavadas en la rama de Ciencias Sociales y Jurídicas son relativamente escasos. Las claves explicativas de esta aparente paradoja radican el reducido tamaño de muchas universidades, que no pueden abordar una oferta de grado dada la dimensión de su plantilla.

Por tanto, se mantienen los vínculos tradicionales con las disciplinas con las que se ha compartido licenciatura hasta hace un par de décadas y con las que se comparte centro en muchos casos (de ahí el peso de la Historia y el Arte en grados como los de las universidades de Oviedo, Alicante, Castilla-La Macha, Extremadura, Lleida o el País Vasco, en los que la formación básica transversal, más que seguir el espíritu de transversalidad del decreto, se rige por una simple y básica estrategia de supervivencia.

La interdisciplinariedad con otras disciplinas de ciencias sociales se logra, en mayor medida, en los grados de La Laguna, la Universidad Complutense de Madrid y Tarragona. Sin embargo, en conjunto, la oportunidad de abrirse y aumentar el intercambio con otras disciplinas mediante la formación básica del nuevo marco de las enseñanzas universitarias no se ha aprovechado plenamente. Los elementos de cambio derivados de la adscripción a rama de conocimiento, pese a ser fruto de un cierto proceso de reflexión, no han conseguido pasar de una cuestión puramente formal debido a unas inercias que siguen muy presentes.

Figura 2

COMPOSICIÓN DE LAFORMACIÓN BÁSICA EN LOS GRADOS DE GEOGRAFÍA

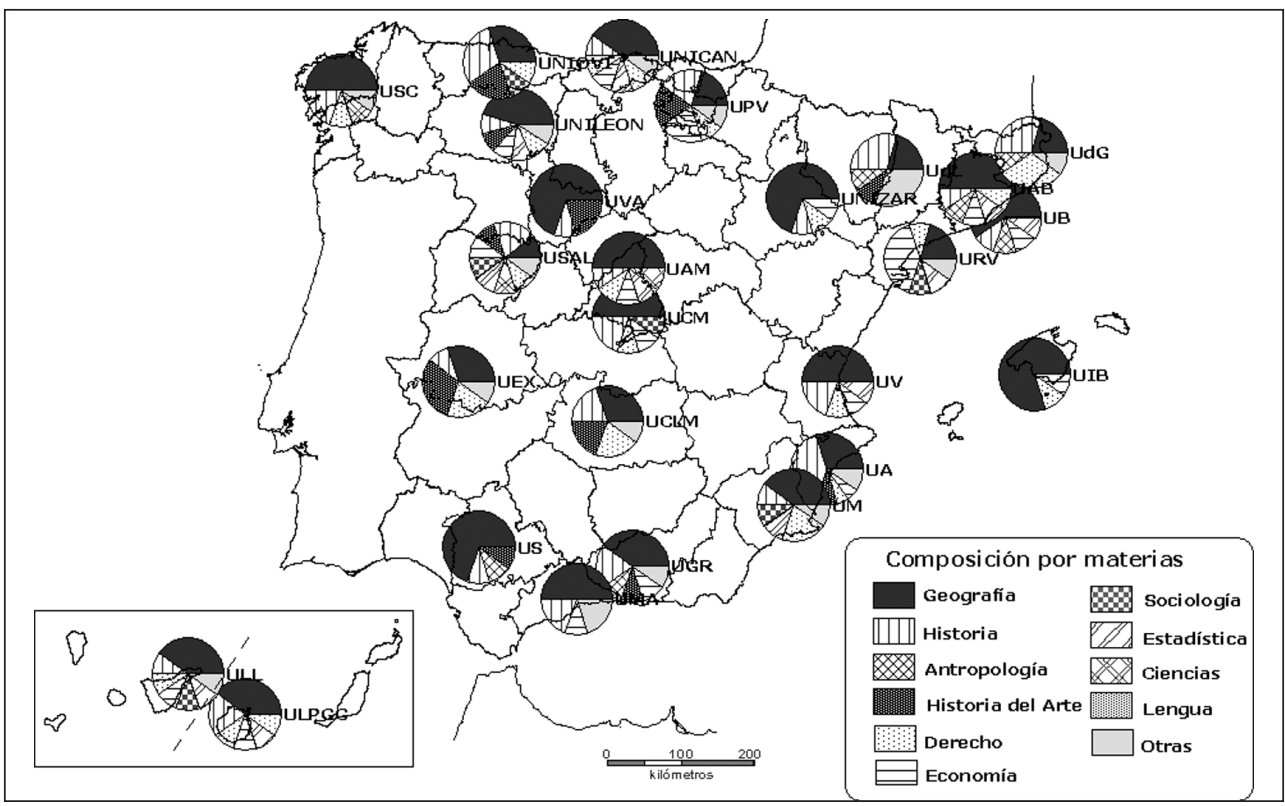

Fuente: Elaboración propia a partir de los grados de Geografía de las diferentes Universidades. 


\subsection{Diversidad y tendencia a reducción en optatividad}

La normativa que regula los grados permite, una vez definida la formación básica, un margen relativamente amplio para la organización de las enseñanzas, por lo que las universidades pueden, en función de sus recursos y del carácter de la titulación, estructurar con bastante libertad materias obligatorias y optativas. Las directrices de cada una de las universidades no responden a las mismas pautas y por ello en algunos casos se ha dejado libertad a los departamentos para realizar propuestas y organizar la optatividad de sus grados, mientras que en otros casos, la optatividad ha sido objeto de una regulación que fijaba las ratios entre el total de créditos optativos a cursar por el alumnado y el total que se podía ofertar en el catálogo de la titulación.

En razón del número de créditos optativos a cursar en las diversas titulaciones de grado (Fig. 3), aparecen diferencias notables entre titulaciones con una optatividad reducida (Alicante, con 18 créditos registra el mínimo y en otras cinco titulaciones se sitúa en 24 créditos) y algunas titulaciones con optatividad elevada (las tres universidades andaluzas junto a la Autònoma de Barcelona tienen 60 o más créditos optativos). El valor medio de la optatividad se sitúa en un valor próximo a 40 créditos ECTS, pero se ha de indicar que esta optatividad incluye, en algunos casos, la oferta de prácticas externas y que, de este modo, es más limitada de lo que se observa a primera vista. Ello supone un elemento de aparente cambio con relación a las licenciaturas, en las que la optatividad - entre optativas propias y créditos de libre elección - llegaba a representar casi la mitad del plan de estudios.

Figura 3

OFERTA TOTAL DE CRÉDITOS OPTATIVOS EN LOS GRADOS DE GEOGRAFÍA

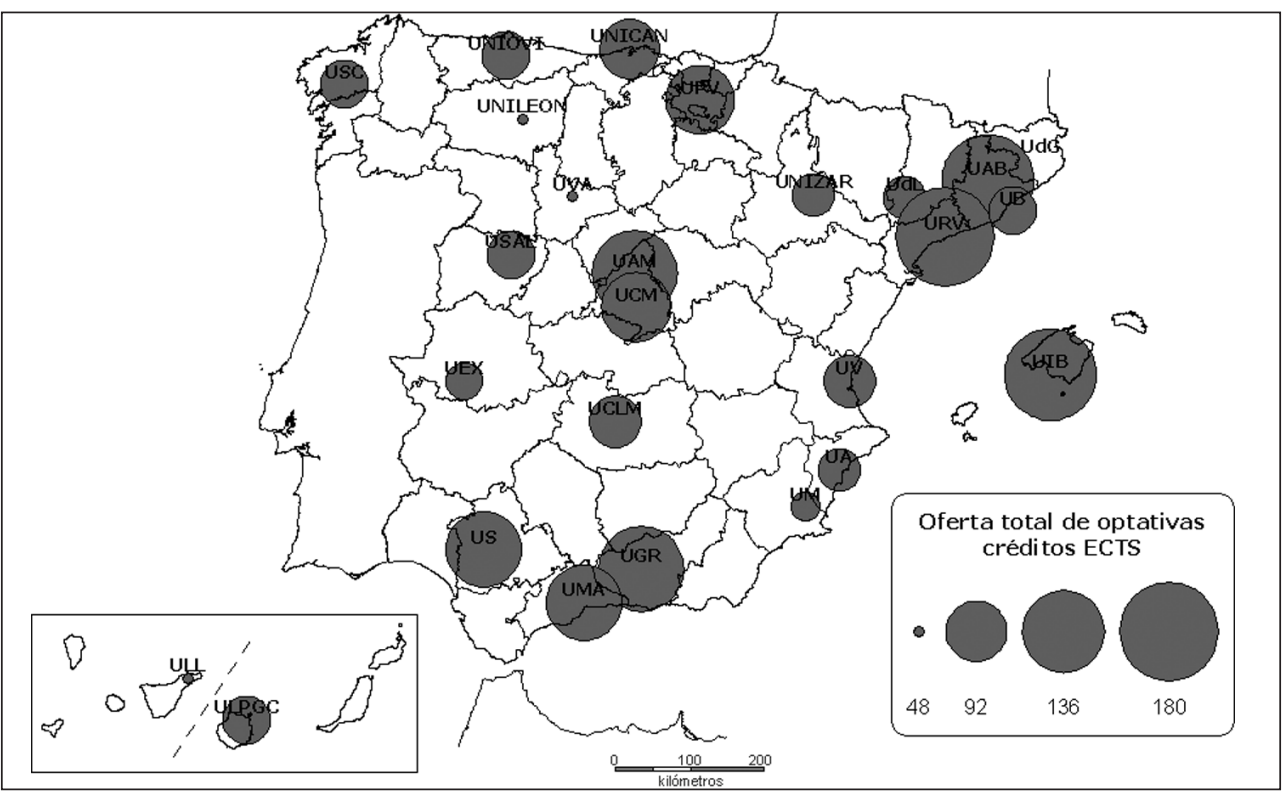

Fuente: Elaboración propia a partir de los grados de Geografía de las diferentes Universidades. 
La ubicación de las optativas es otra de las cuestiones a valorar en este análisis. Dado que en muchos de los grados tiene un peso relativo pequeño (en torno al $15 \%$ ) se ha optado por una arquitectura que prima la obligatoriedad en los tres primeros cursos, de manera que los créditos optativos suelen estar situados en el último de los cursos de grado. Es más, incluso cuando se ofrece la posibilidad de cursar optativas en el tercer curso, se trata de una oferta claramente decantada favor del cuarto curso. Dos cuestiones más a revisar en el análisis de la optatividad son la ratio entre el número de créditos previstos en el plan de estudios y la oferta total optativa del catálogo y la existencia de itinerarios para definir una cierta especialización o intensificación. Con relación a las ratios de optatividad, lo más generalizado es disponer una ratio entre dos y tres por uno (un catálogo que prevé entre dos y tres veces el número de créditos que debe cursar el alumnado). Sin embargo, se constata la diversidad de situaciones, con algunos grados que cuenta con una ratio superior a tres por uno (Illes Balears, Rovira i Virgili, Lleida o Alicante, si bien computan como optativas asignaturas que proceden de la oferta de otras disciplinas, como Historia, Economía o Turismo).

El tema de los itinerarios guarda relación con las limitaciones derivadas de la reducción de la optatividad por lo que, en caso de existir, cuenta con un despliegue reducido. La oferta de itinerarios - la opción de los minors ha sido empleada muy puntualmente en algún caso como la Rovira i Virgili-, más que para ampliar la oferta de optativas ha sido utilizada como elemento articulador u organizativo de la optatividad, lo que se debe señalar como positivo.

Finalmente, cabe introducir la cuestión de si la optatividad está orientada en el mismo sentido que el título que da nombre al grado y en este sentido de si se ofertan optativas relacionadas con la Ordenación del Territorio como elemento innovador. Una rápida revisión permite señalar que ciertamente, en algunas titulaciones se ha depurado la oferta que existían en las licenciaturas y se contemplan asignaturas con denominaciones que parecen coherentes con las nuevas orientaciones socioprofesionales de la Geografía; pero, por otro lado, se consolida - o en ocasiones se recupera - la orientación hacia la formación de profesorado - no formalizada de manera explícita - que permite una imbricación con otras disciplinas de Humanidades, más allá del nominal reacomodo en el campo de las Ciencias Sociales y Jurídicas.

\subsection{EL TRABAJO FINAL DE GRADO Y LAS PRÁCTICAS EXTERNAS: PROYECCIÓN PRÁCTICO- APLICADA DE HABILIDADES Y DESTREZAS EN EL ÁMBITO SOCIOPROFESIONAL}

La normativa establece que todos los grados han de tener un Trabajo Final, obligatorio, pero concede un margen muy amplio en cuanto al número de créditos de éste, pudiendo establecerse entre los 6 y los 30 créditos. En los grados de Geografía no hay grandes variaciones en cuanto al trabajo final. La mitad de los grados plantean trabajos finales de 12 créditos, y casi la otra mitad trabajos de 6 créditos (Fig. 4).

Sólo hay dos excepciones, Lleida con 7,5 créditos por ajuste del número de créditos totales y, sobre todo, el grado de la Universitat Rovira i Virgili de Tarragona, con un trabajo final de 18 créditos. No puede decirse que exista una pauta característica que explique la dimensión de la asignatura porque tanto universidades grandes como pequeñas optan por 12 créditos y la misma diversidad se observa en que decidieron trabajos de 6 créditos. Se trata, 
Figura 4

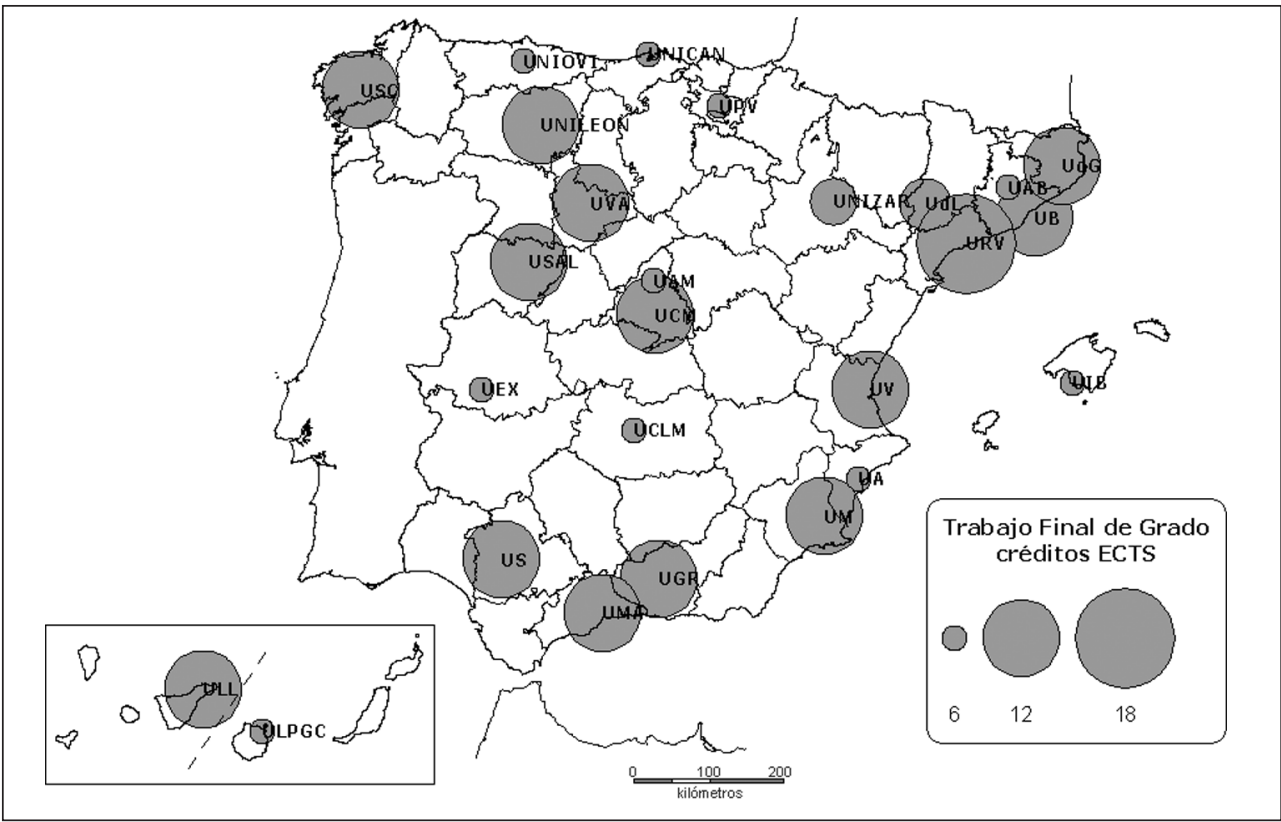

Fuente: Elaboración propia a partir de los grados de Geografía de las diferentes Universidades.

pues, más de estrategias de cada Departamento que de motivaciones como la disponibilidad de recursos docentes, por ejemplo.

En todo caso, lo que sí es cierto es que los departamentos de Geografía afrontan el reto de hacer de estos trabajos finales no tanto un compendio de conocimientos de lo que se ha estudiado durante el resto del grado sino, sobre todo, unos instrumentos de proyección de habilidades y destrezas orientadas al ámbito profesional. La apuesta por Trabajos Finales de 12 créditos no debería de ser una manera de evitar ofertar otras materias, sino el resultado del convencimiento de que los alumnos han de realizar un trabajo de envergadura para poner a punto esas destrezas y habilidades de cara al trabajo profesional.

Por su parte, las prácticas externas son opcionales para los grados, y éstos pueden introducirlas con carácter obligatorio u optativo, pudiendo variar entre los 6 y los 60 créditos. La asunción del interés de las prácticas hace que tan sólo tres de los veintiséis grados analizados no contemplan prácticas externas dentro de su plan de estudios. Por tanto, es un hecho relevante el que la práctica totalidad de departamentos coinciden en la importancia de las prácticas curriculares de cara a la formación de los geógrafos como profesionales. Incluso en los casos en los que los alumnos van a optar por una vía más académico-científica, tales prácticas externas, bien organizadas y estructuradas, y planteadas en tareas que realmente contribuyan a la formación práctica de los alumnos en los grados de Geografía, constituyen una pieza fundamental de cara a la proyección laboral y profesional. 
El análisis de la distribución de las prácticas externas en función del número de créditos arroja resultados similares a los señalados en el caso del Trabajo Final (Fig. 5): más de la mitad plantean prácticas de 6 créditos, y el resto prácticas de 12 créditos o más (el máximo en la Universidad de Las Palmas, con 18 créditos). No se constata correlación clara entre la dimensión en créditos de las prácticas externas y el tamaño de la Universidad.

Figura 5

PRÁCTICAS EXTERNAS: CRÉDITOS Y CARÁCTER OPTATIVO U OBLIGATORIO

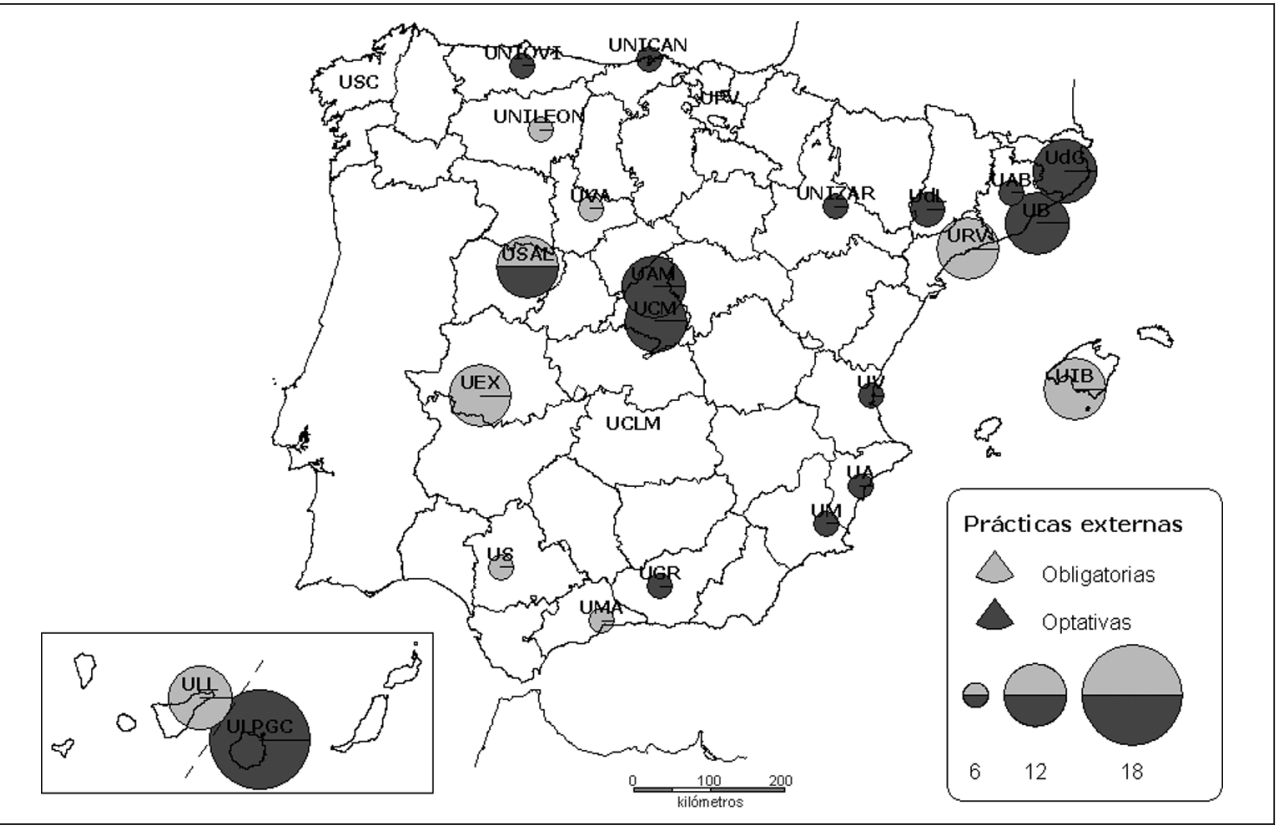

Fuente: Elaboración propia a partir de los grados de Geografía de las diferentes Universidades.

En cambio, debe señalarse como elemento significativo la obligatoriedad u optatividad de las prácticas externa, ya que si uno de los objetivos de los grados en general, y de los de Geografía en particular, es la formación de los alumnos en habilidades y destrezas, además de conocimientos, en el marco de un perfil profesional bien definido (principalmente en torno a la ordenación del territorio), sería esperable que éstas tuviesen un carácter obligatorio, independientemente de que fueran de 6 o 12 créditos. Y sería de esperar que los departamentos estuviesen dispuestos a hacer un esfuerzo por estructurar unas prácticas externas claramente orientadas a ese perfil profesional y de carácter obligatorio. Sin embargo, poco más de un tercio de los grados plantean las prácticas como obligatorias $\mathrm{y}$, tal vez no por casualidad, en su mayor parte con 12 créditos (grados de Geografía de Extremadura, Girona, Illes Balears, La Laguna, Rovira i Virgili o Salamanca).

Es cierto - no obstante - que algunos departamentos han planteado las prácticas externas en el marco de una elevada oferta de optativas, muchas de ellas a su vez con una importante 
componente práctica. Sin embargo, en otros casos, prácticas externas optativas (y además, con pocos créditos) hacen pensar en posiciones cuando menos cómodas y hasta insuficientemente comprometidas con la formación práctica de los estudiantes. El principal reto en este sentido es superar un problema ya clásico como es el desconocimiento e indefinición que muchas empresas e instituciones tienen de los geógrafos y las funciones que éstos pueden realizar. En este ámbito queda, todavía, una importante labor a realizar desde los departamentos de Geografía, para consolidar ese perfil profesional y que éste sea más fácilmente y mejor identificado desde el entorno socio-profesional externo al mundo universitario.

\section{DE LA TEORÍA A LA PRÁCTICA: LA IMPLANTACIÓN DE LOS NUEVOS GRADOS EN GEO- GRAFÍA}

El diseño de las nuevas titulaciones ha supuesto una etapa de reflexión sobre el papel de la Geografía y las funciones que los geógrafos deben/pueden desempeñar en las sociedades actuales, dando lugar a propuestas de grados que son, en parte, un reflejo de estos cambios pero, también, un exponente de los intereses de los colectivos implicados en la docencia de las titulaciones. No obstante, una vez concretada la arquitectura de los títulos, cobra especial relevancia el seguimiento del proceso de implantación (especialmente el análisis de la matrícula de nuevo ingreso), así como el estudio del perfil del alumnado. A la espera de que los primeros graduados finalicen sus estudios (únicamente la reducida primera cohorte de Lleida acabado el grado), cabe realizar un primer balance del paso de los grados que se vertieron en las memorias a los grados que se nutren de nuevos alumnos, del papel a las aulas.

\subsection{La implantación de los nuevos grados: ¿hacia un resurgimiento?}

Como es sabido, las licenciaturas en Geografía experimentaron un deterioro continuo en las cifras de matriculación de nuevo ingreso (Fig. 6). Este paulatino descenso en el número de alumnos dejó, en algunos casos, a los nuevos grados en una franja de relativo peligro antes incluso de su implantación. De hecho, más de un grado se materializó tras un largo debate en torno a su viabilidad, tras una trayectoria de declive continuado en la primera década del siglo XXI.

La evolución de matriculados de nuevo ingreso arroja cifras alentadoras: pese a distar del número registrado en el curso 1998/99, la implantación de los grados, prácticamente generalizada en el curso 2009/10 supone consolidar una tendencia al alza que se refuerza en el curso 2010/11. Así pues, la idea de que el grado supone una recuperación significativa, un relanzamiento de la titulación está claramente avalada por las estadísticas disponibles (tabla 2).

El crecimiento es evidente en la mayoría de casos y espectacular en muchas universidades que pasan a duplicar el número de sus alumnos de nuevo ingreso. Se debe valorar, por tanto, muy positivamente estos datos que ponen de manifiesto la buena acogida de las propuestas tanto en los casos en que se vinculan con la Ordenación del Territorio (un nuevo atractivo, sin duda para el alumnado) como en las que mantienen un perfil más tradicional con un grado en Geografía. 


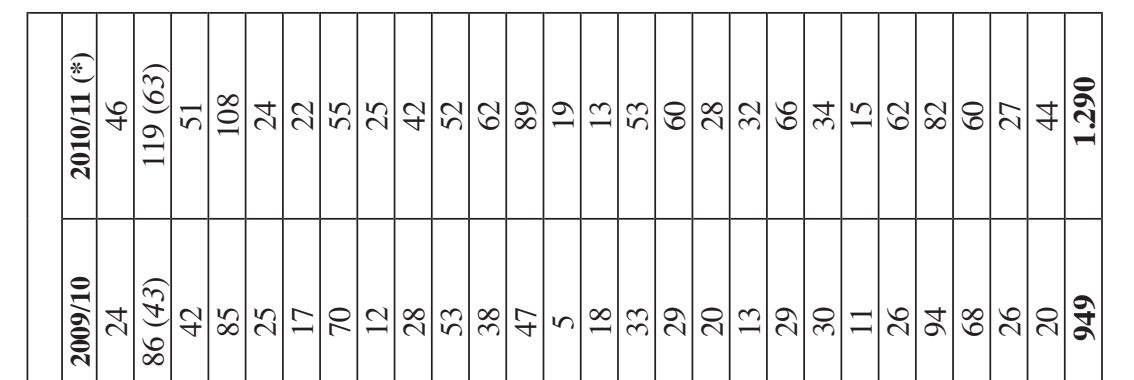

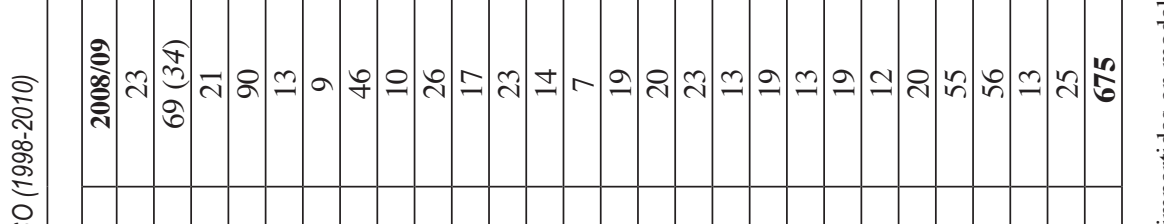

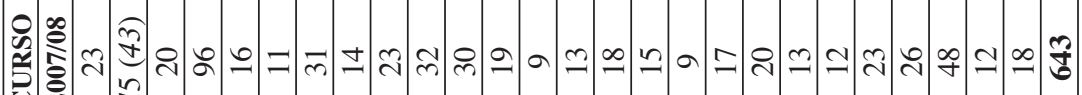









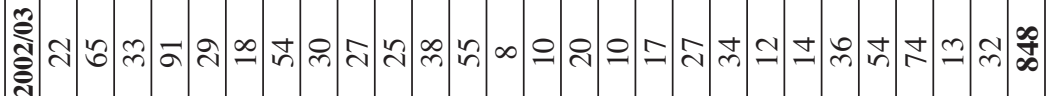

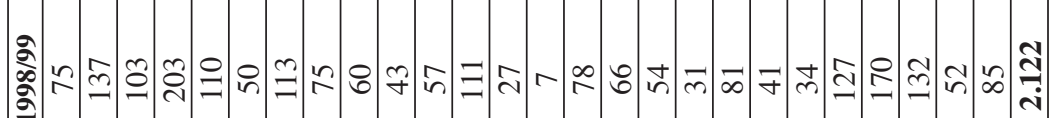

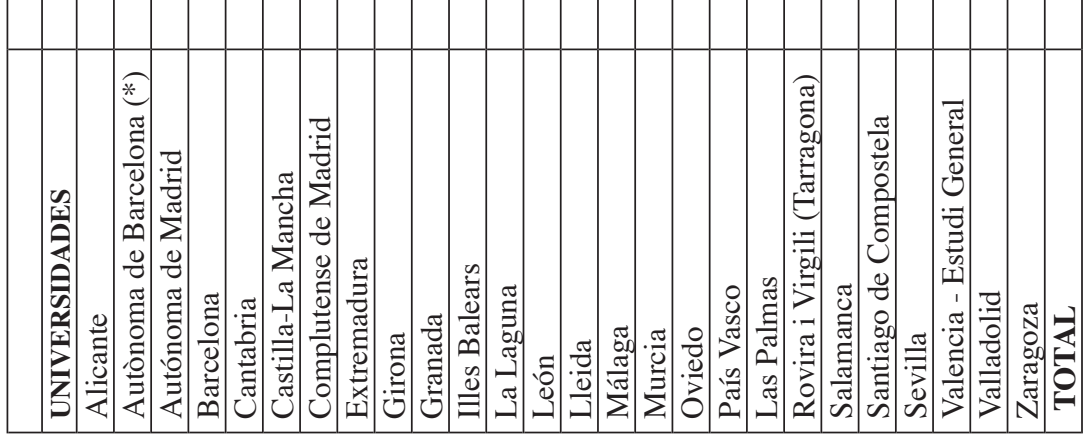


Figura 6

MATRICULADOS DE NUEVO INGRESO EN LICENCIATURAS Y GRADOS DE GEOGRAFÍA (1998/99=100)

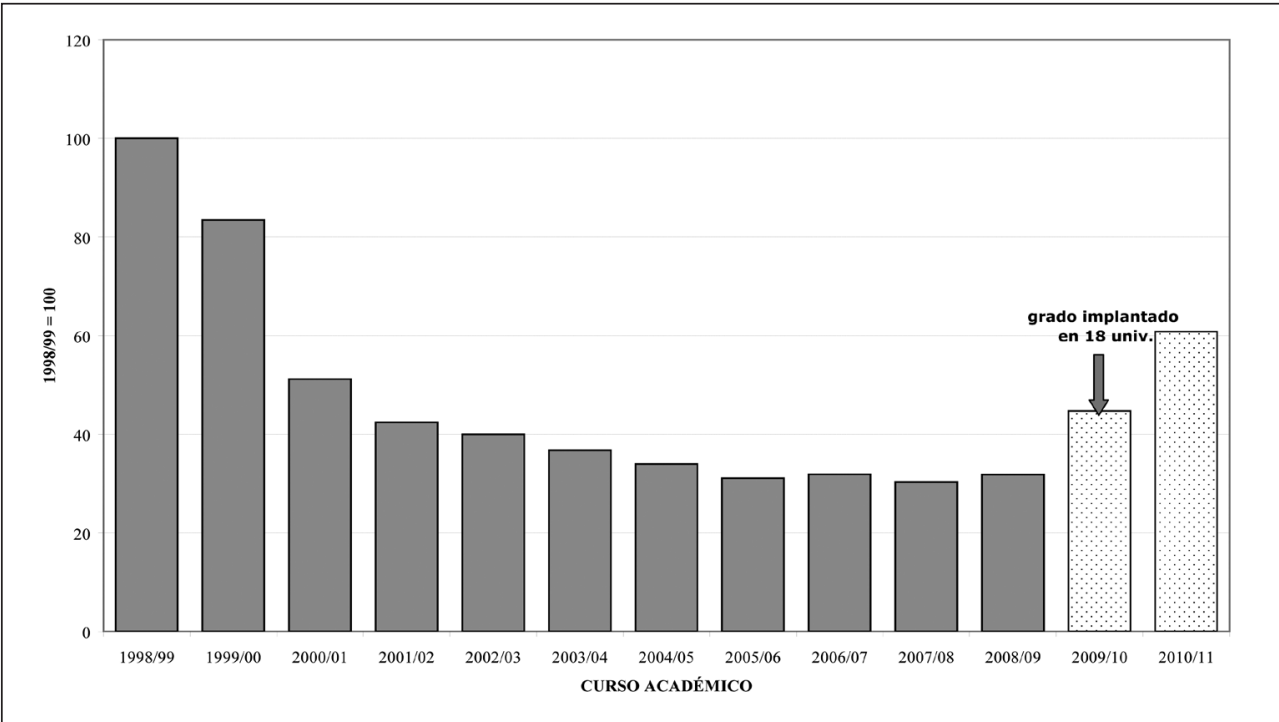

Fuente: Elaboración propia a partir de Estadísticas del Ministerio de Educación y Encuesta AGE-Colegio de Geógrafos.

Figura 7

UNIVERSIDADES SEGÚN TAMAÑO DE LA COHORTE DE NUEVO INGRESO 1998/99 - 2010/11

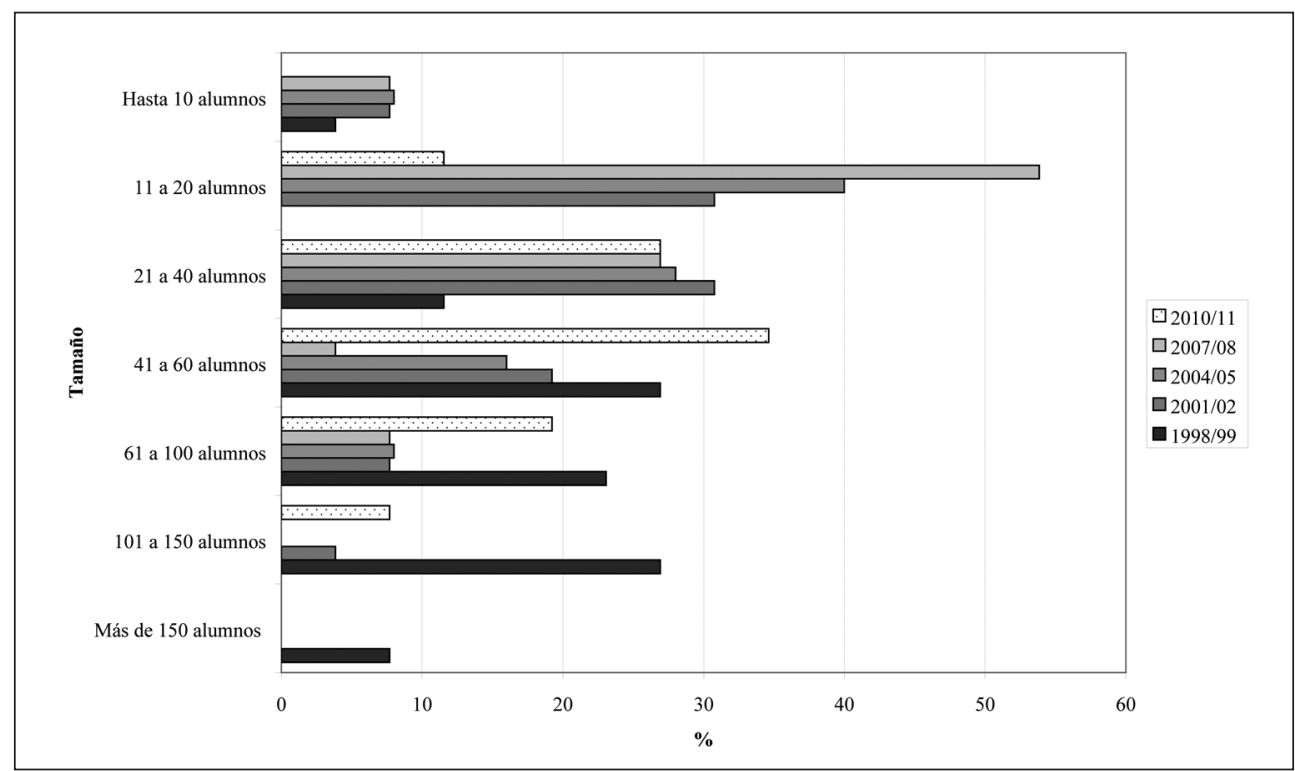

Fuente: Elaboración propia a partir de Encuesta de grados de AGE-Colegio de Geógrafos (2011). 
సै



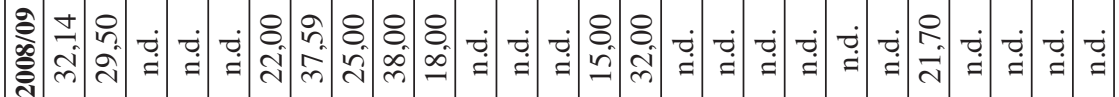

迹

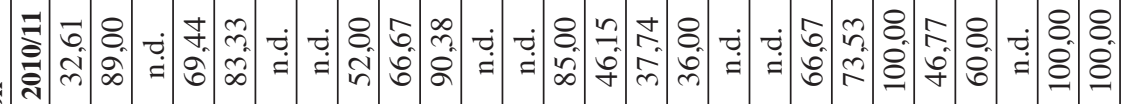

要

嵌

넌



:

๓ 㟧

중

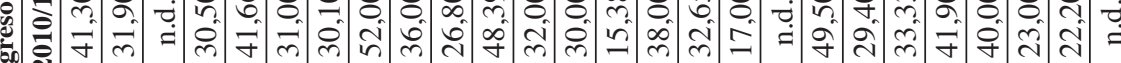
운

8



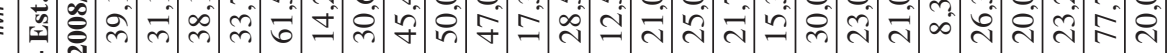

虫

ริm ڤ

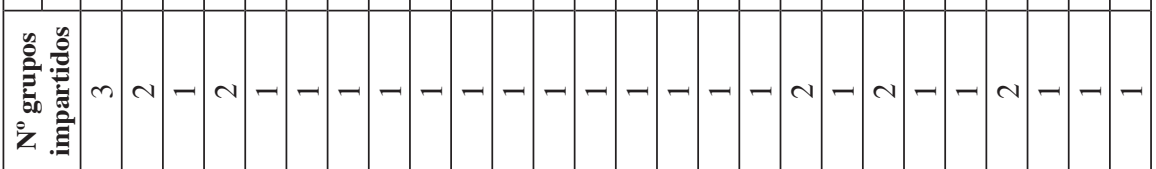

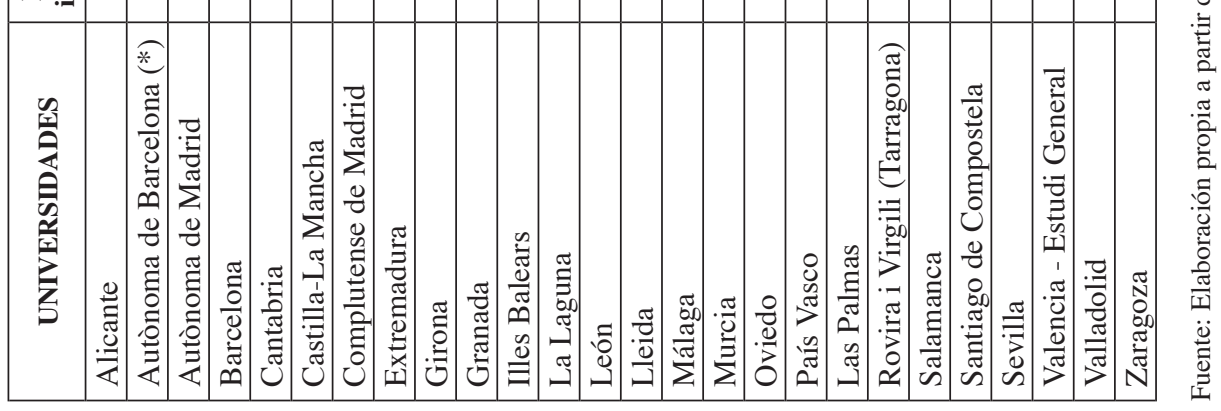


No obstante y tras la felicitación colectiva por este éxito de la disciplina, cabe introducir algunas cuestiones para la reflexión, como el papel desempeñado por la coyuntura económica general que ha colaborado, en mayor o menor medida, en el incremento de matrícula de jóvenes que no encuentran opciones claras en el mercado laboral. Ello conduce a plantearse si la recuperación es un hecho estructural tras la reforma de planes de estudios que ahora contemplan en mayor medida la profesionalización o si, por el contrario, se trata de una etapa pasajera debido a las dificultades de inserción laboral.

\subsection{Nueva titulación ¿nuevos perfiles?}

La nueva orientación del grado y la atracción de una cifra más elevada de alumnado conducen a la necesidad de revisar los perfiles de los alumnos, con objeto de constatar si las modificaciones han hecho variar los patrones de alumnado que cursaba la licenciatura. La escasez de datos y la limitada trayectoria de la experiencia en grados suponen un obstáculo para poder sistematizar los análisis. Con todo, a la luz de los datos recopilados por la encuesta elaborada por la AGE y el Colegio de Geógrafos, se pueden apuntar algunas primeras tendencias que habrá que revisar con mayor aparato estadístico.

La reducción de alumnado en el transcurso del primer decenio del siglo XXI se acompañó, con frecuencia, de una progresiva masculinización del alumnado (tabla 3). Entre 1998 y 2008, 17 de las 26 universidades que impartían licenciaturas de Geografía redujeron su proporción de mujeres en el alumnado de nuevo ingreso. La recuperación de la matrícula revierte esta tendencia en muchos casos, de manera que la implantación del grado supone una mayor feminización.

En segundo lugar, y tras el análisis de la demanda en primera opción (tabla 3), cabe constatar que es frecuente que el aumento de matriculados vaya parejo a un descenso de esta demanda. Ello genera nuevos retos: llegan más alumnos pero menos vocacionales y por tanto es preciso desarrollar estrategias para la fidelización, para motivar interés en aquéllos que no pensaban cursar un grado en Geografía (Geografía y Ordenación del Territorio) en primera instancia. Los datos correspondientes al abandono son insuficientes para detectar tendencias claras pero la experiencia de otras etapas apuntan a que el abandono se puede incrementar (y así lo muestran algunos datos recogidos) en paralelo a la disminución de la demanda en primera opción, tal y como se ha señalado.

\section{RETOS Y REFLEXIONES}

Tres son los grandes retos que la sigue teniendo la Geografía española. A corto plazo, la fidelización del alumnado; a corto-medio plazo, avanzar en la cultura del seguimiento y el próximo proceso de acreditación; y a medio-largo plazo hacer del Grado (y los masters) un instrumento de proyección profesional.

\section{a) La fidelización del alumnado}

Se ha señalado y hemos visto que con los títulos de grado se había experimentado una recuperación en la matrícula de nuevo ingreso, superando incluso los niveles del curso 2000- 
2001. Pero los departamentos de Geografía en España no podemos quedarnos con el buen dato de esta recuperación. Tenemos que conseguir una cierta estabilidad, que dote de sostenibilidad al Grado en las diferentes universidades. Porque se corre el riesgo, pese a que los títulos de Geografía hayan sido atractivos en estos primeros años, de caer en una nueva senda descendente, y lo que sería peor, el desprestigio y/o marginalización al que ello podría estar asociado. Por ello, con el proceso de implantación ya prácticamente finalizado, tenemos que llevar a cabo un análisis serio y riguroso sobre las tasas de rendimiento, de eficiencia académica, de abandono, y de graduación y, en su caso, estudiar las causas y sobre todo poner en marcha lo antes posible de los mecanismos para contrarrestar esos posibles resultados. Y recordemos que la labor de fidelizar al alumnado implica el diseño de estrategias y acciones en tres ámbitos. En primer lugar, el referido al propio título de Grado, su diseño, su orientación, los recursos disponibles, etc. En segundo lugar, el propio profesorado, actuando sobre nuestra disponibilidad y actitud de cara al proceso de mejora de la calidad, y si fuese necesario, también sobre nuestra preparación y capacitación profesional. En este sentido es de destacar que, por lo general, las nuevas generaciones de profesores universitarios mantienen actitudes más abiertas y receptivas a los cambios y a todo lo que implica la mejora de los procesos de calidad. Y en tercer lugar habría que continuar actuando en el ámbito de la proyección profesional y la empleabilidad, que constituye en realidad otro de los grandes retos de la Geografía española en el inmediato futuro.

\section{b) La mejora de la calidad en el contexto de la acreditación de los títulos}

Los grados constituyen una apuesta por la calidad. Más allá de los esfuerzos que cada universidad o departamento pudiera hacer para mejorar la calidad docente, la implantación de los Grados implica un proceso de acreditación de los mismos (a los 5 años de la implantación), como recoge el Real Decreto 1393/2007. Este texto insistía en la importancia de los sistemas de calidad, y junto a la verificación inicial de los títulos oficiales, establece un sistema de evaluación periódica que concluirá con la acreditación de los mismos, que habrá de ser renovada cada cierto tiempo. En 2010 un nuevo texto resalta la importancia, además, del seguimiento en la ejecución de los títulos. Por tanto, el proceso de seguimiento y acreditación se convierten en la medida del éxito en el proceso de adaptación al EEES. Aunque ahora nos lleva por la vía de la imposición, es evidente que los mecanismos de control y seguimiento son necesarios, en cualquier caso, y ello implica que desde el sistema universitario en general, y desde los departamentos de Geografía en particular, avancemos en la cultura del seguimiento y la evaluación, asumiendo estas tareas como algo inherente al proceso formativo.

Tres son las cuestiones clave a tener en cuenta para el seguimiento: el desarrollo de la titulación (es decir, hasta qué punto se está haciendo todo lo necesario para asegurar que la implantación de los títulos se produce de acuerdo con lo establecido); el logro de metas (es decir, hasta qué punto en la titulación se están alcanzando los objetivos previstos y se están obteniendo resultados considerados como positivos); y por último el grado de relevanciautilidad de la titulación para los estudiantes (es decir, hasta qué punto la titulación es útil para los graduados y les permite integrarse laboral y socialmente). 
Para medir todo ello se plantean un conjunto de indicadores, básicamente de tres tipos, de entrada a los títulos, de salida de éstos, y los referidos a los recursos disponibles como apoyo al aprendizaje. Respecto a la entrada a los títulos los indicadores se refieren sobre todo a los alumnos, como sus vías de acceso, las calificaciones de entrada o las plazas ofertadas, pero también sus características personales (edades y sexos, si realizan estudios a tiempo completo o parcial), familiares (nivel de estudios de los padres, etc.) y sociales (residencia, desplazamientos, etc.). Como se observa los indicadores de entrada constituyen básicamente un trabajo de recopilación y sistematización de la información administrativa de los estudiantes.

Los indicadores referidos a los recursos disponibles como apoyo al aprendizaje tampoco son complejos en esencia. Aquí se incluye el tipo de profesorado (según categorías), su cualificación en cuanto a méritos docentes y de investigación; la distribución de horas de clase (clases teóricas o magistrales, seminarios, laboratorios, tutorías, etc.), y tamaño de los grupos; la utilización que el profesorado y los alumnos hacen del campus virtual (a partir del material disponible, el número de accesos y volumen de descargas, etc.); los métodos de evaluación empleados (exámenes, ensayos, exposiciones, prácticas, etc.); las prácticas externas que realizan los alumnos y su movilidad; las actividades de orientación para la profesión y la inserción laboral que realiza la universidad, centro o departamento; la satisfacción del profesorado respecto del proceso docente; y la tradicional satisfacción de los estudiantes respecto del profesorado y la titulación.

Por último, los indicadores de salida son los más complejos y probablemente los que van a ser analizados con más detenimiento a la hora de la acreditación. Se refieren básicamente a los resultados personales de los estudiantes y a sus resultados académicos. Los resultados personales pretenden valorar tanto la inserción laboral (a través de un seguimiento de los graduados durante cierto tiempo) como el grado de satisfacción de los empleadores. De esta forma el grado de inserción laboral nos estaría dando pistas precisas sobre el logro de las competencias previstas así como la relación entre los objetivos de aprendizaje y los requisitos profesionales, todo ello teniendo en cuenta la situación en el mercado laboral. En su caso, la valoración de los trabajos finales de los alumnos y su orientación serían igualmente un indicador de los resultados personales, si bien los más importantes se refieren a la inserción laboral.

Por su parte, los resultados académicos son también importantes y probablemente más fáciles, a corto plazo, de valorar de forma más precisa y sistemática. Aquí se contemplan cuatro tasas, de rendimiento, abandono, eficiencia y graduación.

$\checkmark$ La tasa de rendimiento hace referencia a la proporción de créditos ordinarios que el alumno ha superado respecto de los matriculados. Una tasa baja podría interpretarse como mayores dificultades de los estudiantes en sus estudios, o bien que éstos no han seguido una estrategia adecuada a la hora de diseñar la secuencia de materias. En todo caso, nos estaría avisando de la necesidad de un cierto asesoramiento en cómo el estudiante organiza su plan de estudios, teniendo en cuenta, en su caso, las capacidades personales y las dificultades que pudiera tener respecto de las diferentes materias del grado.

$\checkmark$ La tasa de eficiencia es la diferencia entre el número de créditos realmente matriculados con los que teóricamente deberían haber matriculado, es decir, se contempla el número de materias que han debido matricularse dos o más veces. Es un indicador del 
exceso de créditos que un estudiante precisa para obtener el título de grado. Es decir, cuanto más nos alejamos de 100 menor es la eficiencia, y mayor la dificultad del plan de estudios. Detectar en qué materias se produce este exceso de créditos matriculados debería conducirnos a tomar medidas concretas sobre ellas y su docencia.

$\checkmark$ La tasa de abandono es la proporción de estudiantes que abandonan los estudios cada año y en cada uno de los tres primeros cursos. Cuando se detectan porcentajes elevados de abandono el departamento debe analizar con detenimiento los motivos de ese abandono, cuándo se produce y qué materias, en su caso, están implicadas, y tomar las medidas adecuadas para corregir estos resultados.

$\checkmark$ La tasa de graduación es la proporción de estudiantes que finalizan en el periodo de cinco años (los cuatro previstos más uno adicional, considerado como razonable) con respecto a los matriculados inicialmente. Es un indicador del grado de ajuste entre el diseño inicial del plan de estudios y su implantación. Cuando los valores se alejan del $100 \%$ estaríamos ante un plan estudios desajustado respecto de la formación previa y de las capacidades esperadas de los estudiantes, ante mayores dificultades generales en el conjunto de las materias, ante una planificación inadecuada, o en su caso ante procedimientos de evaluación del aprendizaje inadecuados.

\section{c) Los nuevos títulos como instrumentos de proyección profesional y empleabilidad}

Los títulos de Grado, y los masters directa o indirectamente vinculados a ellos, son (o han de ser) sobre todo instrumentos para la empleabilidad de nuestros estudiantes. Recordemos que en el contexto socioeconómico en el que nos encontramos una de nuestras principales responsabilidades es responder a las necesidades sociales a través de la formación y el aprendizaje que procuramos a nuestros estudiantes. El Libro Blanco recogía ya un análisis tanto de potenciales empleadores de lo que vendría a ser un graduado en Geografía como de las necesidades de estos potenciales empleadores. Aunque la situación actual es parcialmente diferente a la que había hace apenas una década (con, por ejemplo, una contracción de la demanda desde las administraciones públicas), se detectaban una serie de nichos de mercado idóneos para la formación geográfica (ANECA, 2004; Colegio de Geógrafos, 2008; Tulla, 2010).

Con los títulos de Grado en la mano, con el proceso de implantación prácticamente concluido, podemos ya hacernos algunas preguntas. Por ejemplo, si estamos respondiendo adecuadamente a esas necesidades; si los Grados (y los Master) son instrumentos adecuados para formar y capacitar adecuadamente a futuros profesionales; si su diseño es el necesario para alcanzar los objetivos perseguidos (obviamente contando con el actual contexto restrictivo en cuando a recursos); o si las capacidades con las que contamos el profesorado son las necesarias y, en su caso, en qué medida en cada departamento hemos diseñado (o pensamos hacerlo) estrategias de organización-ordenación docente y de especialización-capacitación que permitan una respuesta más eficaz a las nuevas necesidades.

También hay otras consideraciones específicas a tener en cuenta y que influyen de manera decisiva en la calidad y adecuación del producto que estamos ofreciendo, nuestros títulos, y en cómo este es útil para nuestros estudiantes a la hora de avanzar en su inserción profesional. Efectivamente, el colectivo de geógrafos no podemos dejar de lado otras reflexiones, 
como el papel de la transversalidad, de la optatividad, las prácticas externas, el trabajo final y los masters. Una primera reflexión está referida al posicionamiento de la Geografía respecto de otras disciplinas, sobre todo las Ciencias Sociales. Muchos departamentos apostaron, en sus títulos, por una cooperación con otras disciplinas más allá de las tradicionales Historia e Historia del Arte. Es momento de preguntarse si esa cooperación es real y se está traduciendo en avances hacia una formación más sólida y hacia una verdadera transversalidad en las ramas de conocimiento, si habría que reforzarla, reorientarla o, en su caso, eliminarla. Y en el caso de la optatividad, hay que valorar si es realmente un instrumento de especialización y en qué medida los diseños de cada título responde a una clara estrategia formativa o bien es la plasmación parcial (como desgraciadamente ha ocurrido en algún momento) de la especialización investigadora del profesorado.

Habrá que valorar igualmente en qué medida las prácticas externas y el trabajo final están siendo instrumentos de desarrollo y proyección de habilidades y destrezas para el ejercicio profesional, o si, en el caso del trabajo final, lo estamos concibiendo como un compendio de conocimientos. Pero es igualmente importante analizar qué estrategias de inserción profesional se han diseñado en los departamentos y qué papel tienen tanto las prácticas externas como el trabajo final en esa estrategia, en su caso. Y esto implicaría un trabajo serio de búsqueda, seguimiento y mejora de las prácticas externas en contacto directo con potenciales empleadores.

Finalmente, aunque apenas se han abordado aquí los postgrados y masters, tras varios años de implantación de los títulos oficiales hay que preguntarse sobre la viabilidad real que tienen los masters de o vinculados a Geografía en cada universidad (variable en función de una serie de factores, entre ellos el tamaño de la universidad y del área potencial de mercado). En todo caso parece evidente que hemos de avanzar hacia una oferta más competitiva en el medio plazo, y ello probablemente implicará un proceso de racionalización y, en el mejor de los casos, de eficaz articulación entre las diferentes ofertas. Y habrá que tomar decisiones, casi inmediatas, si este esfuerzo de articulación ha de hacerse pensando en la especialización profesional de los geógrafos (lo cual nos conduciría, por ejemplo, a masters interuniversitarios de Geografía); o bien si ha de hacerse pensando en las problemáticas a abordar, en las necesidades sociales, y aquí habríamos de avanzar hacia masters interdisciplinares, inter o universitarios. Obviamente todo lo relacionado con la cooperación en torno a los masters oficiales exige una fuerte voluntad de entendimiento y de superación de la burocracia administrativa, y aquí las estructuras universitarias han de ser sin duda mucho más permeables.

Agradecimientos: Los autores agradecen a los departamentos de Geografía sus respuestas a la encuesta que en su día se canalizó a través del Colegio Profesional de Geógrafos y AGE en 2011, y que han permitido completar la información necesaria para el presente trabajo.

\section{REFERENCIAS BIBLIOGRÁFICAS}

ANECA (2004): Libro Blanco para el diseño del Título de Grado en Geografía y Ordenación del Territorio. Madrid, Aneca. 
ARROWSMITH, C., BAGOLY-SIMÓ, P., FINCHUM, A., ODA, K., y PAWSON, E. (2011): «Student employability and its implications for geography curricula and learning practices». Journal of Geography in Higher Education, Vol. 35, nº 3, 365-377.

BURRIEL DE ORUETA, E., (2004a): «La base formativa común en la enseñanza universitaria de la Geografía en España». Boletín de la Asociación de Geógrafos Españoles, ${ }^{\circ}$ $38,47-70$.

BURRIEL DE ORUETA, E., (2004b): «La licenciatura de Geografía en la Universidad Española actual», en Comité Español de la UGI: Aportación Española al XXX Congreso de la Unión Geográfica Internacional. Madrid, Comité Español de la UGI, 9-46.

COLEGIO PROFESIONAL DE GEÓGRAFOS (2008): Perfiles profesionales de los geógrafos en España. Disponible en:

http://www.geografos.org/images/stories/interes/perfiles/Perfiles_profesionales.pdf

CONWAY-GÓMEZ, K., WILLIAMS, N., ATKINSON-PALOMBO, C., AHLQVIST, O., KIM, E., y MORGAN, M. (2011): «Tapping geographys potential for synergy with creative instructional approaches». Journal of Geography in Higher Education, Vol. 35, ${ }^{\circ}$ 3, 409-423.

ESPARCIA, J., SÁNCHEZ AGUILERA, D. (2009): «Innovación e inercia en el proceso de adaptación al EEES: el caso de los nuevos títulos de Grado en Geografía» VI Foro sobre la evaluación de la calidad de la educación superior y de la investigación. Vigo, 8-11 Septiembre. Comunicación-póster.

ESPARCIA, J., SÁNCHEZ AGUILERA, D. (2010): «Retos e inercias en el proceso de adaptación de la Geografía al EEES en España». VI Congrés Internacional de Docència Universitària i Innovació (CIDUI). Nous espais de qualitat en l'educació superior. pp. 1-32.

HENNEMANN, S., \& LIEFNER, I. (2010): «Employability of german geography graduates: The mismatch between knowledge acquired and competences required». Journal of Geography in Higher Education, Vol. 34, n 2, 215-230.

PRIETO, A. (2010): Entrevista personal a Antonio Prieto, Presidente del Colegio Profesional de Geógrafos, Valencia, 8 Febrero de 2010.

ROONEY, P., KNEALE, P., GAMBINI, B., y KEIFFER, A. (2006): «Variations in international understandings of employability for geography». Journal of Geography in Higher Education, Vol. 30, n $1,133-146$.

TULLA, A. (2010): «Los nuevos planes de estudio de los títulos de grado en Geografía adaptados al modelo del Espacio Europeo de Educación Superior (EEES)», Estudios Geográficos, Vol. LXXI, n $268,319-338$.

WHALLEY, W. B., SAUNDERS, A., LEWIS, R. A., BUENEMANN, M., y SUTTON, P. C. (2011): «Curriculum development: Producing geographers for the 21 st century». Journal of Geography in Higher Education, Vol. 35, n 3, 379-393. 
